\title{
Thromboembolic injury and systemic toxicity induced by nicotine in mice
}

\author{
Mohamed A. Fahim ${ }^{1}$, Abderrahim Nemmar ${ }^{1}$, Suhail Al-Salam², Subramanian Dhanasekaran², \\ Mohamed Shafiullah ${ }^{3}$, Javed Yasin ${ }^{4}$ and Mohamed Y. Hasan ${ }^{3}$ \\ ${ }^{1}$ Department of Physiology, Faculty of Medicine and Health Sciences, United Arab Emirates University, Al Ain, UAE \\ ${ }^{2}$ Department of Pathology, Faculty of Medicine and Health Sciences, United Arab Emirates University, Al Ain, UAE \\ ${ }^{3}$ Department of Pharmacology, Faculty of Medicine and Health Sciences, United Arab Emirates University, Al Ain, UAE \\ ${ }^{4}$ Department of Internal Medicine, Faculty of Medicine and Health Sciences, United Arab Emirates University, Al Ain, UAE
}

\begin{abstract}
Nicotine is involved in the pathogenesis of hematological and cardiopulmonary diseases. However, the understanding of the pathophysiological mechanisms underlying these undesirable effects is unclear. Cigarette smoking, nicotine gums and patches are common sources for nicotine ingestion. We have investigated the nicotine's effect on cerebral microvessel thrombosis and systemic toxicity. Mice received either nicotine $(1 \mathrm{mg} / \mathrm{kg}$, i.p.) or saline (control), once a day for 21 days. Briefly, after bolus intravenous fluorescein injection, a photo insult of cerebral microvessel was done. The platelet aggregation in microvessels was video recorded and analyzed. In conjunction, the plasma levels of superoxide dismutase (SOD), lactate dehydrogenase (LDH), liver enzymes, creatinine and blood urea nitrogen (BUN), and histopathological studies were carried out. Our results revealed a significant prothrombotic effect following nicotine exposure. Significant decrease in SOD indicates the occurrence of oxidative stress involved in the tissue damages and increase in the LDH emphasize the systemic toxicity. Substantial rise in the liver aspartate aminotransferase (AST) and alanine aminotransferase (ALT) levels were observed. Lungs histology showed intra-vascular hemorrhagic infarction with necrosis, macrophage and neutrophils infiltration. Liver histology showed intravascular thrombosis and portal inflammation. We conclude that the sub-acute nicotine exposure causes an increase in thrombosis in cerebral microvessels and systemic, hepatic and pulmonary toxicity.
\end{abstract}

Key words: Nicotine - Thrombosis - Systemic toxicity - Liver function - Histopathology

\begin{abstract}
Abbreviations: ALT, alanine aminotransaminase; AST, aspartate aminotransaminase; BUN, blood urea nitrogen; GGT, gamma glutamyl transferase; HCT, hematocrit; HGB, hemoglobin; LDH, lactase dehydrogenase; MCV, mean corpuscular volume; PLT, platelets; SOD, superoxide dismutase; WBC, white blood cells.
\end{abstract}

\section{Introduction}

Nicotine, one of the primary noxious components of cigarette smoke is a naturally occurring alkaloid found in many plants. The principal sources of nicotine exposure are through the use of tobacco, nicotine containing gum, and nicotine re-

Correspondence to: Mohamed A. Fahim, Department of Physiology, Faculty of Medicine and Health Sciences, United Arab Emirates University, P.O. Box 17666, Al Ain, UAE

E-mail: m.fahim@uaeu.ac.ae placement therapies (Yildiz et al. 1998). Nicotine is known to have disparaging effects on the cardiopulmonary system which includes lung damage, acute cardiac syndrome, disturbance in the platelet contents and its aggregation, renal circulatory changes, the alteration of the liver functions (Hanna 2006) and is most widely associated with cancer (Bernhard et al. 2003; Rahman and Laher 2007). Nicotine plays major role in increasing the blood pressure (Lindenblatt et al. 2007) and the atherosclerosis (Lee and Cooke 2011). Experimentally, an association between atherosclerosis and venous thrombosis has been reported (Prandoni et al. 2003). 
Inflammation and thrombosis are now recognized to be interdependent processes and complement each other (Nemmar et al. 2007, 2009, 2011). Upregulation of the expression of various proteins such as basic fibroblast growth factor, tumor necrosis factor- $\alpha$ and plasminogen activator inhibitor- 1 are caused by nicotine (Lau et al. 2006). Suffusion with nicotine was found to enhance P-selectin-dependent leukocyte rolling in a murine model of cerebral microcirculation (Yong et al. 1997). P-selectin played major role in enhancing the peripheral thrombogenicity after lung inflammation is mediated by platelet-leukocytes activation (Nemmar et al. 2007).

Nicotine administration to rats through the high tar tobacco cigarette smoke significantly influence the epithelial cell layer thickening with increased lung permeability as measured by histopathological examination (Miller et al. 1996). Nicotine worsens liver fibrosis largely in chronic liver disease patients (Soeda et al. 2012). Weights of the liver increased to 60 days treatment of nicotine with the appearance of cartilaginous cells in the heart and deposition of adipose around the portal vein in the liver (Iranloye and Bolarinwa 2009).

Nicotine-induced contraction of coronary arteries is related to superoxide anion-mediated degradation of nitric oxide. Treatment of rabbit aortas with an oxygen free radical scavenger attenuated cigarette smoke impairment of arterial relaxation (Hanna 2006). Thrombin is a potent platelet agonist, and activated platelets may amplify thrombin generation. Circulating platelets do not normally adhere to the endothelial cells of the vascular wall or aggregate, unless when triggered due to injury to the endothelium is attained. Smoking is linked to such thromboembolic events (Hioki et al. 2001). We have recently demonstrated that acute (1 week) exposure to nicotine causes thrombotic complication in mice (Fahim et al. 2011). However, a relationship between the subacute nicotine exposition to platelet aggregation and its influence with the oxidative stress involving superoxide dismutase (SOD) and the lactate dehydrogenase (LDH), a marker of cytotoxicity, level has not been established.

Therefore, the aim of this study is to assess the subacute effects of nicotine on thrombotic events directly from the cerebral microcirculation, the level of SOD, LDH, liver enzymes including alanine aminotransferase (ALT), aspartate aminotransferase (AST), gamma glutamyl transferase (GGT) and circulatory cells. Moreover, histopathological studies on lung, liver, heart and kidney were also evaluated.

\section{Materials and Methods}

This project was reviewed and approved by the Institutional Review Board of the United Arab Emirates University, Fac- ulty of Medicine and Health Sciences, and experiments were performed in accordance with protocols approved by the Institutional Animal Care and Research Advisory Committee.

Adult male mice, TO strain, aged 8 months old, weighing 30-35 g from a colony maintained at our animal house facility were used. Animals were group-housed in a temperature maintained $\left(22 \pm 2^{\circ} \mathrm{C}\right)$ and a light/dark cycle $(12 / 12 \mathrm{~h})$ environment. They had access to standard pelleted diet and water ad libidum. Except for their intended treatment all animals were handled in the same manner. All animal treatment procedures met both the National Institute of Health (NIH) and Institutional Animal Ethics Committee guidelines.

Nicotine (Sigma Chemicals, MO, USA) aqueous (40\%) solution of dose $1 \mathrm{mg} / \mathrm{kg}$ animal weight was dissolved in normal saline and injected intraperitoneally (i.p.) for three weeks every day. Normal saline solution (vehicle) was injected in the same manner to the control group. At the end of the treatment, the animals were subjected to our experiments.

\section{Thromboembolic microcirculation study}

In vivo pial cerebral microvessel thrombogenesis was assessed as previously described (Al Dhaheri et al. 1995; Nemmar et al. 2009). At the end of the treatment, the mice ( $n=$ 9) were anaesthetized with urethane $(25 \%, 0.1 \mathrm{ml} / 10 \mathrm{~g}$ b.w., i.p.), trachea was intubated and tail vein was cannulated for the intravenous injection. On the anesthetized mice craniotomy was performed and the cerebral microvessels were exposed on the left side of the head with a microdrill. The duramater was removed carefully. The body temperature was stabilized at $37^{\circ} \mathrm{C}$ with the help of an infrared lamp and monitored with a rectal thermoprobe (Physitemp Model RET-3). Artificial cerebro-spinal fluid (ACSF) at the composition of (mM): $\mathrm{NaCl} 124, \mathrm{KCl} 5, \mathrm{NaH}_{2} \mathrm{PO}_{4} 3$, $\mathrm{CaCl}_{2} 2.5, \mathrm{MgSO}_{4} 2.4, \mathrm{NaHCO}_{3} 23$, and glucose $10,(\mathrm{pH}$ 7.3) was superfused to keep the cranium moist. Any signs of trauma to the exposed blood vessels in mice were excluded from the study. The closed circuit camera (CCTV) was used to record and analyze the blood vessel circulation. The CCTV system consisted of the microscope (Olympus, $\mathrm{BH}-2$ ), a video fluorescence microscope camera (JVC, TK$890 \mathrm{E}$ ), a video recorder (JVC, BR-S600E) and a television monitor (JVC, TM-1500PS). Sodium fluorescein (2\%, 0.1 $\mathrm{ml} / 10 \mathrm{~g}$ b.w.) was injected via the tail vein. It was allowed to circulate in the body for $30 \mathrm{~s}$ before mercury light was switched on.

The combination of mercury light exposure (44,000 lux candles $/ \mathrm{cm}^{2}$ ) and fluorescein dye produces a photo chemical insult and injures the endothelium of the microvessel lumen. This in turn, causes platelets to adhere at the sites of endothelial damage and then aggregate. The fluorescence of 
aggregates was readily visible as they adhered to the damaged endothelium. Platelet aggregates grow in size until complete vascular occlusion (Al Dhaheri et al. 1995). The time when the first observable aggregate appeared (time to first aggregate) and until full vascular occlusion (time to flow stop) in both venules and arterioles were measured in seconds. The time of aggregate growth was calculated as the difference between the time to stop the flow and the time to form a first aggregate. All events were videotaped for analysis and measurement of microvessel diameter changes.

\section{Measurement of SOD in plasma}

Determination of SOD, a free radical scavenging enzyme, was carried out in plasma to assess the possible effect of nicotine on oxidative stress. The SOD activity was measured using a commercial kit (Cayman Chemical, Ann Arbor, MI, USA) (Nemmar et al. 2010).

\section{Measurement of LDH in plasma}

The LDH was measured using commercial kits (Sigma Chemical, St. Louis, MO, USA) which determine the conversion of lactate to pyruvate in the presence of LDH with an equivalent lessening of NAD. The formation of NADH from the above reaction can show a difference when measured in absorbance at $340 \mathrm{~nm}$.

\section{Complete blood count analysis}

The mice $(n=10)$ were anesthetized intra-peritoneally with sodium pentobarbital $(45 \mathrm{mg} / \mathrm{kg})$, and then blood was drawn from the inferior vena cava in EDTA (4\%). Part of the blood was used for platelets (PLT) and white blood cells (WBC) counts using an ABX VET ABC Hematology Analyzer with a mouse card (ABX Diagnostics, Montpellier, France). The remaining blood was centrifuged for 15 min at $4^{\circ} \mathrm{C}$ at $900 \times g$, and the plasma obtained was stored at $-80^{\circ} \mathrm{C}$ pending analysis. In conjunction with the WBC and PLT estimation, red blood cells (RBC), hemoglobin (HGB), hematocrit (HCT) and mean corpuscular volume (MCV) were measured to assess the effect of nicotine in circulatory cells and related parameters.

\section{Measurement of ALT, AST, GGT in plasma}

Determination of ALT, AST and GGT as the markers of liver function tests were performed in plasma. The ALT and AST were measured using standard laboratory methods using LX20 multiple automated analyzer (Beckman Coulter, CA, USA). The GGT, a membrane-bound enzyme involved in the trans-membrane uptake of glutathione and the elevated levels reflect the diseases of the liver, biliary system, and pancreas, was measured using commercial kits (Sigma Chemical, St. Louis, MO, USA).

\section{Measurement of creatinine and blood urea nitrogen in plasma}

The kidney damages occurring due to nicotine were studied by the measurement of plasma creatinine and blood urea nitrogen (BUN). The creatinine and urea were measured spectrophotometrically using commercial kits purchased from Human GmbH (Wiesbaden, Germany).

\section{Histopathology methods}

Tissue samples including lungs, liver, kidney and heart from both the control and nicotine-treated groups have undergone the histopathological processing and were studied for their changes in microscopical structures. The organs were excised, washed with ice-cold saline, blotted with filter paper and weighed. Each organ was cassetted and fixed directly in $10 \%$ neutral formalin for 24 hours, which was followed by dehydration in increasing concentrations of ethanol, clearing with xylene and embedding with paraffin. Sections of $4 \mu \mathrm{m}$ were prepared from paraffin blocks and stained with haematoxylin and eosin. The stained sections were evaluated by the histopathologist that participates in this project using a light microscope (Olympus BX41 with Olympus camera DP71).

\section{Statistical analysis}

All data were statistically analyzed with GraphPad Prism Version 4.01 for Windows software (GraphPad Software Inc., San Diego, CA). Data are expressed as means \pm S.E.M. Comparisons between groups were performed using the unpaired Student's $t$-test; $p<0.05$ was considered to indicate statistical significance.

\section{Results}

\section{Thromboembolic microcirculation study}

Nicotine treatment influences the time required for platelet aggregation and full occlusion in pial microvessels which are summarized in the Fig. 1. Our results showed that for first platelet aggregation time there is no difference in arterioles but a significant difference in venules $(p<0.001)$ when compared to the control animals. However, the time taken for complete flow stop due to aggregation in the arterioles $(p<0.001)$ and venules $(p<$ 0.001 ) were both significant. Data on the first aggregate 


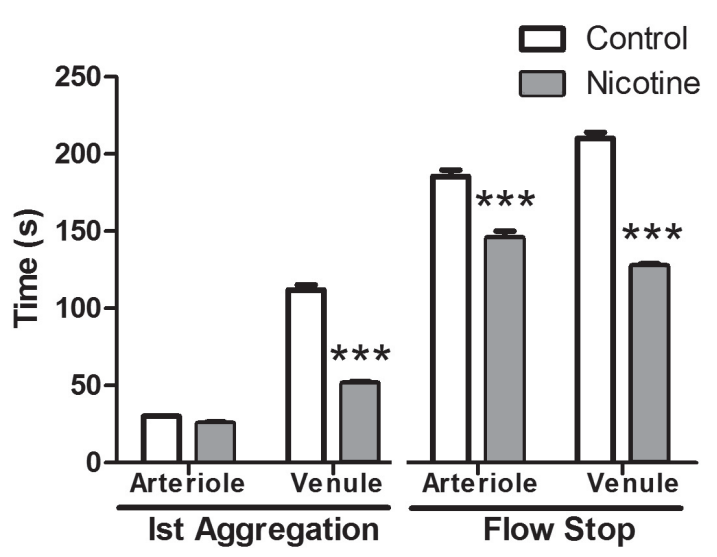

Figure 1. Effect of nicotine treatment on first aggregation and flow stop in both arterioles and venules in cerebral microvessel thrombo-embolic study. The values shown are the mean \pm SEM $(n=9) ;{ }^{* * *} p<0.001$.

in pial arterioles and venules of the nicotine-treated group indicates an increased susceptibility to thrombosis in nicotine-exposed animals. The vascular diameter changes throughout the photochemical insult among both groups were within a very narrow range and no statistically significant differences were present.
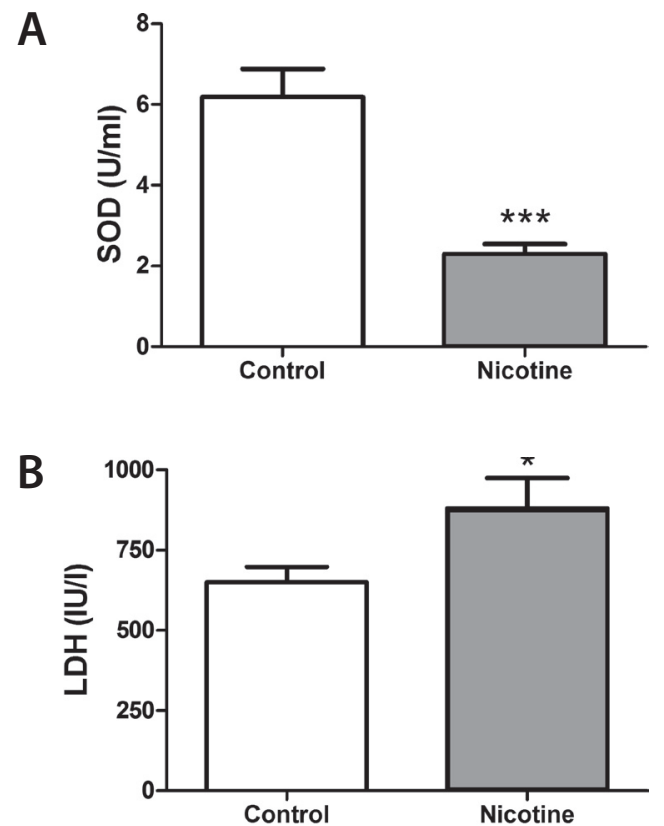

Figure 2. Effect of nicotine treatment on the mouse plasma levels of superoxide dismutase (SOD) (A) and lactate dehydrogenase $(\mathrm{LDH})(\mathbf{B})$. The values shown are the mean $\pm \mathrm{SEM} ;{ }^{\star} p<0.05$, ${ }^{* * *} p<0.001$ compared to control group.
Oxidative stress studies

SOD is an important antioxidant enzyme present in nearly all cells involved in mitochondrial respiration. The plasma level of the SOD was significantly decreased in the nicotine $(p<0.001)$ treated group indicating that SOD in the plasma was markedly depleted by the nicotine inducing oxidative stress (Fig. 2A).

\section{Measurement of LDH}

LDH level in plasma of the nicotine-treated group showed a significant increase when compared to the control group $(p<0.05)$ (Fig. 2B).

\section{Complete blood count analysis}

Our results showed that the WBC and RBC count, hemoglobin level, hematocrit volume, platelet count measured did not differ significantly in the nicotine-treated group compared to the control one (Fig. 3A-E). MCV measure of the average red blood size, which is reported as a standard part of the whole blood count, showed no significant change (Fig. 3F).

\section{Measurement of AST, ALT and GGT}

Liver enzymes namely AST and ALT were significantly altered in the groups of mice which received nicotine. Significant increase in AST $(p<0.05$; Fig. 4A) and ALT ( $p<0.05$; Fig. $4 \mathrm{~B}$ ) levels were observed in the nicotinetreated groups when compared to the controls. Elevated serum GGT activity can be found in diseases of the liver, biliary system, and pancreas. In our studies, the level of GGT increased in the nicotine-treated group but failed to show a statistical difference at the dose tested (Fig. 4C).

\section{Measurement of creatinine and BUN}

The estimation of creatinine and BUN was performed to measure the influence of the nicotine to assess any kidney damage. Our results showed no significant change in the creatinine level and BUN in the nicotine-treated group when compared to the control group at the dose tested (Fig. 5).

\section{Histopathology}

Lung histology: The control saline-treated group shows normal lung morphology and architecture (Fig. 6A). In contrast, the nicotine-treated group shows intra-vascular thrombosis (Fig. 6B), hemorrhagic infarction of the lung with necrosis of inter-alveolar septae (Fig. 6C) with many 
hemosiderin-laden macrophages in the infarcted area (Fig. 6D) and inflammatory cell infiltration with widening of the inter-alveolar septae which consists of polymorphonuclear neutrophils and mononuclear cells (Fig. 6E).

Liver histology: In contrast to the normal liver morphology and architecture in saline-treated group (Fig. 7A) there were foci of intra-vascular thrombosis involving the central hepatic venule in sections of the nicotine-treated group (Fig. 7B,C). The thrombi were tightly adherent to the endothelial surface of the affected terminal hepatic venules. Liver sections of nicotine-treated group show foci of portal inflammation (Fig. 7D). In addition, foci of widening and dilatation of terminal hepatic venules were present (Fig. 7E).

Heart and kidney histology: The heart and kidney sections of both the control and nicotine-treated groups show normal heart morphology and architecture (Fig. 8).
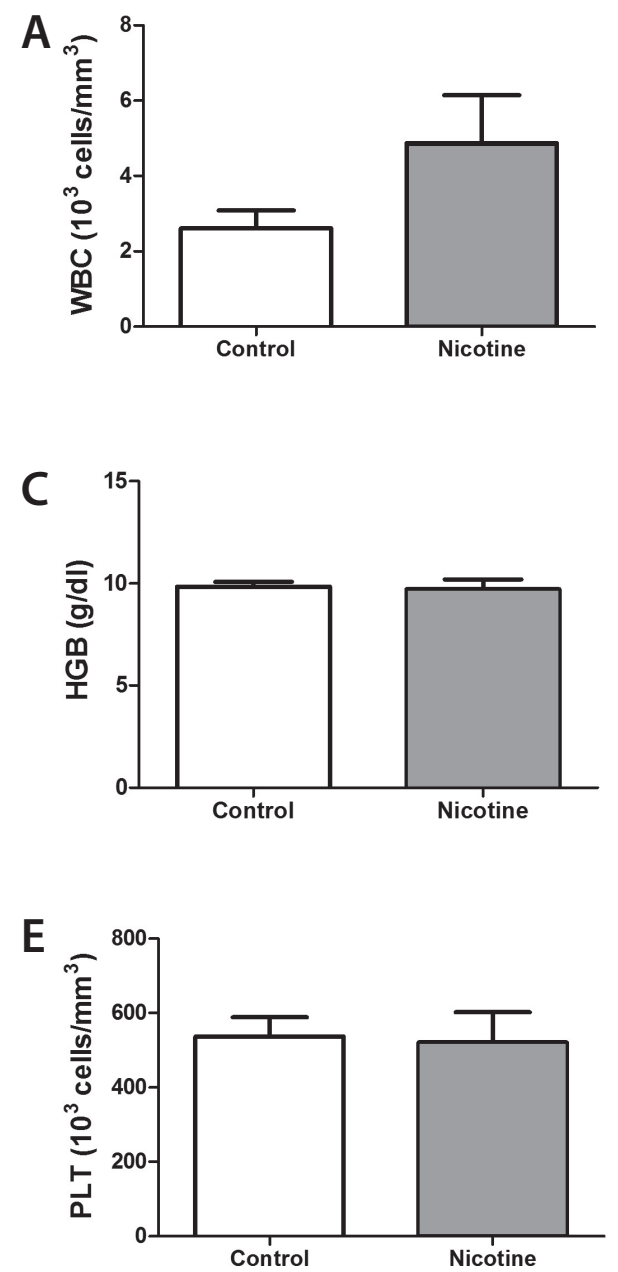

\section{Discussion}

The dose of the nicotine ( $1 \mathrm{mg} / \mathrm{kg} /$ day, i.p., 21 days) selected in the present study was based on various references including a review article (Matta et al. 2006) and Elliott et al. 2004 who reported effects of acutely appplied nicotine already in dose of $1 \mathrm{mg} / \mathrm{kg}$, s.c. and $3 \mathrm{mg} / \mathrm{kg}$, i.v. . The dose used in our study was without any gross behavioral or body changes in mice. Nicotine (1 mg/kg, i.p., once a day for three weeks) caused: 1) rapid aggregation leading to complete blood flow stop in the microvessels, 2) oxidative stress measured as significant reduction of SOD levels, 3) cytotoxicity expressed as increased LDH level, 4) liver toxicity showed an increase in the levels of AST and ALT, 5) an insignificant increase in WBC level without alteration in the RBC, HGB, HCT, PLT and MCV, as well as 6) necrotic hemorrhagic infarction and inflammation in lung and liver.

B
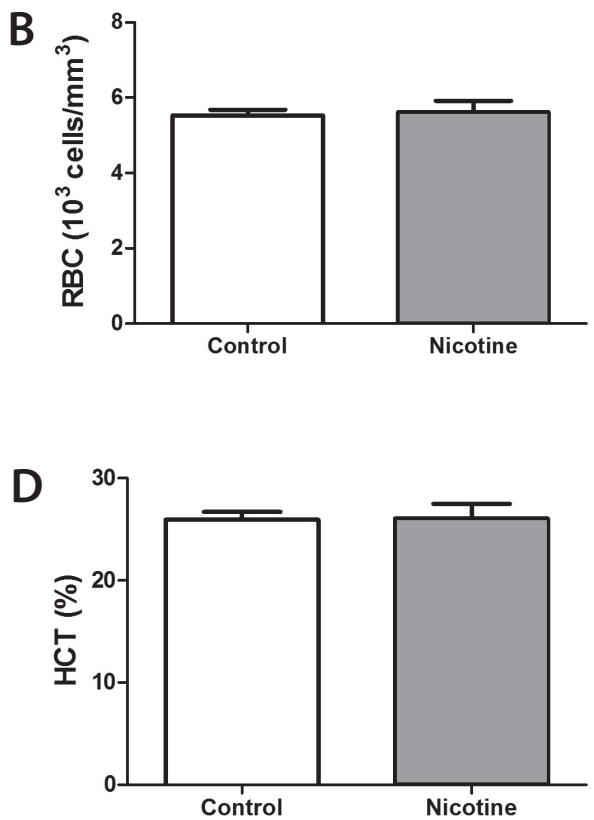

$\mathbf{F}$

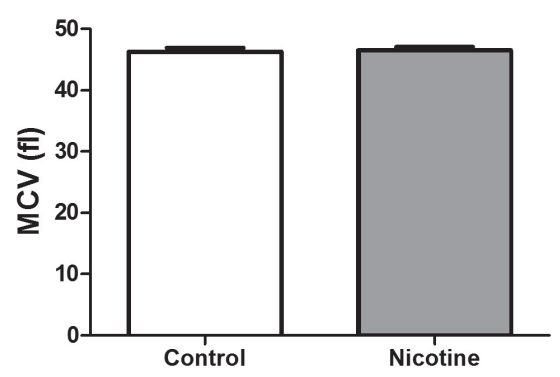

Figure 3. Effect of nicotine treatment in the complete blood cell counts: leucocytes (WBC) (A), erythrocytes (RBC) (B), total hemoglobin (HGB) (C) and hematocrit (HCT) (D), platelets (PLT) (E), mean corpuscular volume (MCV) (F) in mice. The values shown are the mean $\pm \operatorname{SEM}(n=10)$. 

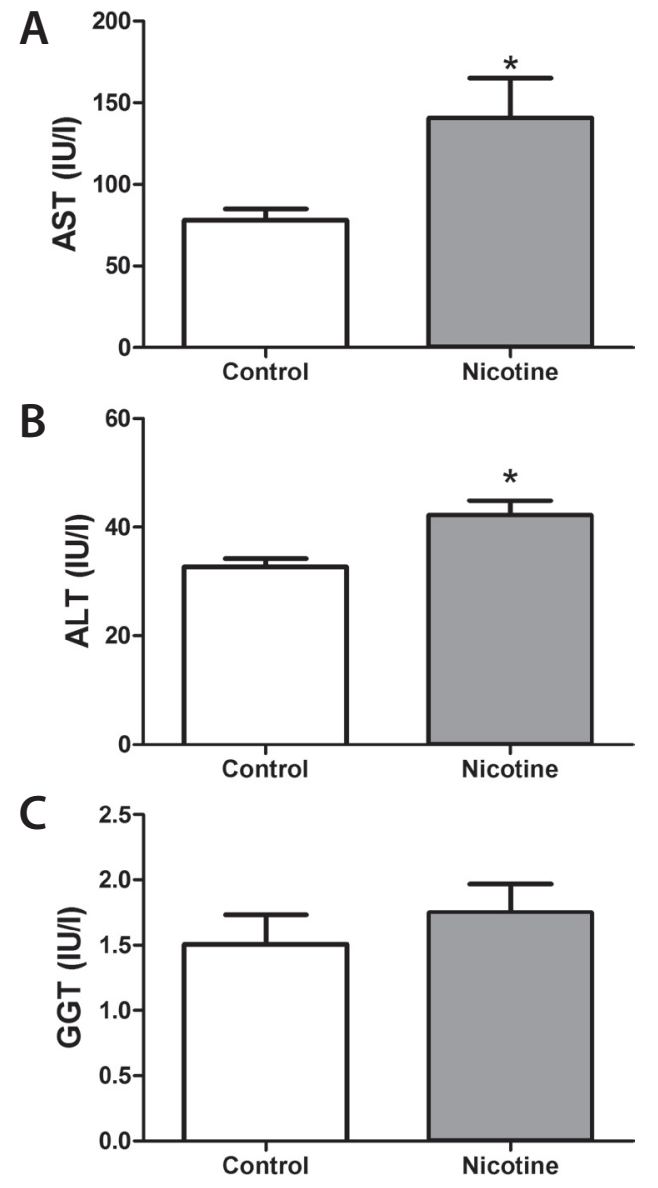

Figure 4. Effect of nicotine treatment on the mouse plasma levels of aspartate transferase (AST) (A), alanine aminotransferase (ALT) (B) and gamma glutamyl transpeptidase (GGT) (C). The values shown are the mean $\pm \operatorname{SEM}(n=13) ;{ }^{*} p<0.05$ compared to control group.

Our kidney function studies, which included creatinine and BUN measurements, did not show a significant change when compared to the control group. Likewise, no histological changes were observed in the kidneys after nicotine exposure.

Because it has widely been accepted that the male species are relatively stable while the female species are limited to few interfering factors such as hormonal changes during the estrous cycles in the results our work was limited to the male gender. Although it is highly significant to include female mice, the effect of the gender differences is currently placed out of the scope of our study and will be addressed in future.

Previous reports which focused on thrombosis describes the nicotine exerting direct effects on endothelial cells and smooth muscle cells, shifting them toward a pro-thrombotic state via induction of tissue factor expression (Cirillo et al.
2006) and via increased platelet dependent thrombogenesis (Hioki et al. 2001). These effects on the vessel wall might explain, at least in part, the deleterious cardiovascular, liver and lung injuries of nicotine. In this study subacute daily dose of nicotine for three weeks influences a quick aggregation and complete blood flow stop in the cerebral arterioles, clearly indicates the adverse effects of nicotine.

On the other hand, the in-vitro platelet aggregability studies by different authors have reported conflicting results following cigarette smoking or nicotine exposure. The platelet aggregation induced by nicotine produced no change (Siess et al. 1982), an increase (Folts and Bonebrake 1982; Renaud et al. 1984; Hung et al. 1995) or a decrease (Foo et al. 1991) in platelet aggregability following smoking. The reason behind the variability of the results on the platelet aggregability is not clearly known. However, the condition of the blood withdrawn from different subjects wherein different health conditions such as coronary artery disease (Hung et al. 1995), exercised heart (Siess et al. 1982), high cigarette smoking (Folts and Bonebrake 1982; Renaud et al. 1984), habitual smoking (Foo et al. 1991) could explain the difference of observations. Discussion on the variability of the aggregability is out of the scope of this study, however, it is evident that acute increased concentrations of nicotine quicken the aggregability inducing thrombogenesis in the circulatory system (Folts and Bonebrake 1982; Renaud et al. 1984; Hung et al. 1995; Hioki et al. 2001; Cirillo et al. 2006).
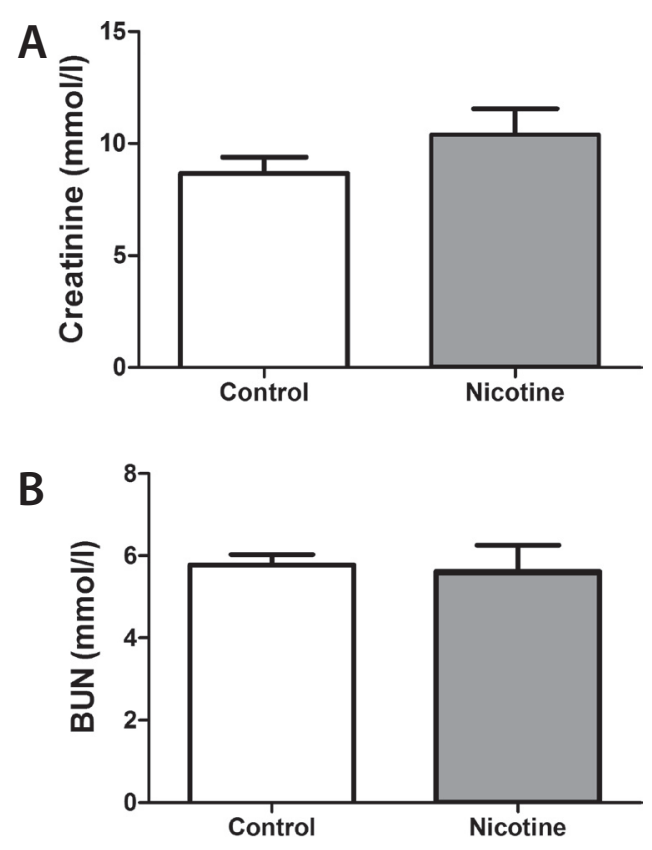

Figure 5. Effect of nicotine treatment on the mouse plasma levels of creatinine (A) and blood urea nitrogen (BUN) (B). The values shown are the mean $\pm \operatorname{SEM}(n=13)$. 
Smoking is considered to induce oxidative stress which in turn causes atherosclerosis and other systemic toxicities (Niki 2004; Singh and Jialal 2006). Oxidative stress arises from an imbalance between the production of free radicals and physiological antioxidant capability. The supplementation of the antioxidants like vitamin $\mathrm{C}$ or $\mathrm{E}$ has been reported to provide health benefits against the platelet aggregation reduced by acute exposure to nicotine in mice (Fahim et al.
2011) and cardiovascular diseases (Steiner 1999). Nicotine causes injury to the vascular endothelium, produces superoxide anions, reduces both production and bioavailability of nitric oxide (NO), increases both production and release of endothelin, causes endothelial dysfunction, thrombosis, infarction, coronary artery disease, stroke and death (Sabha et al. 2000; Fang et al. 2003, 2006). In a recent report (Arrick and Mayhan 2007) the rats with acute and chronic treatment

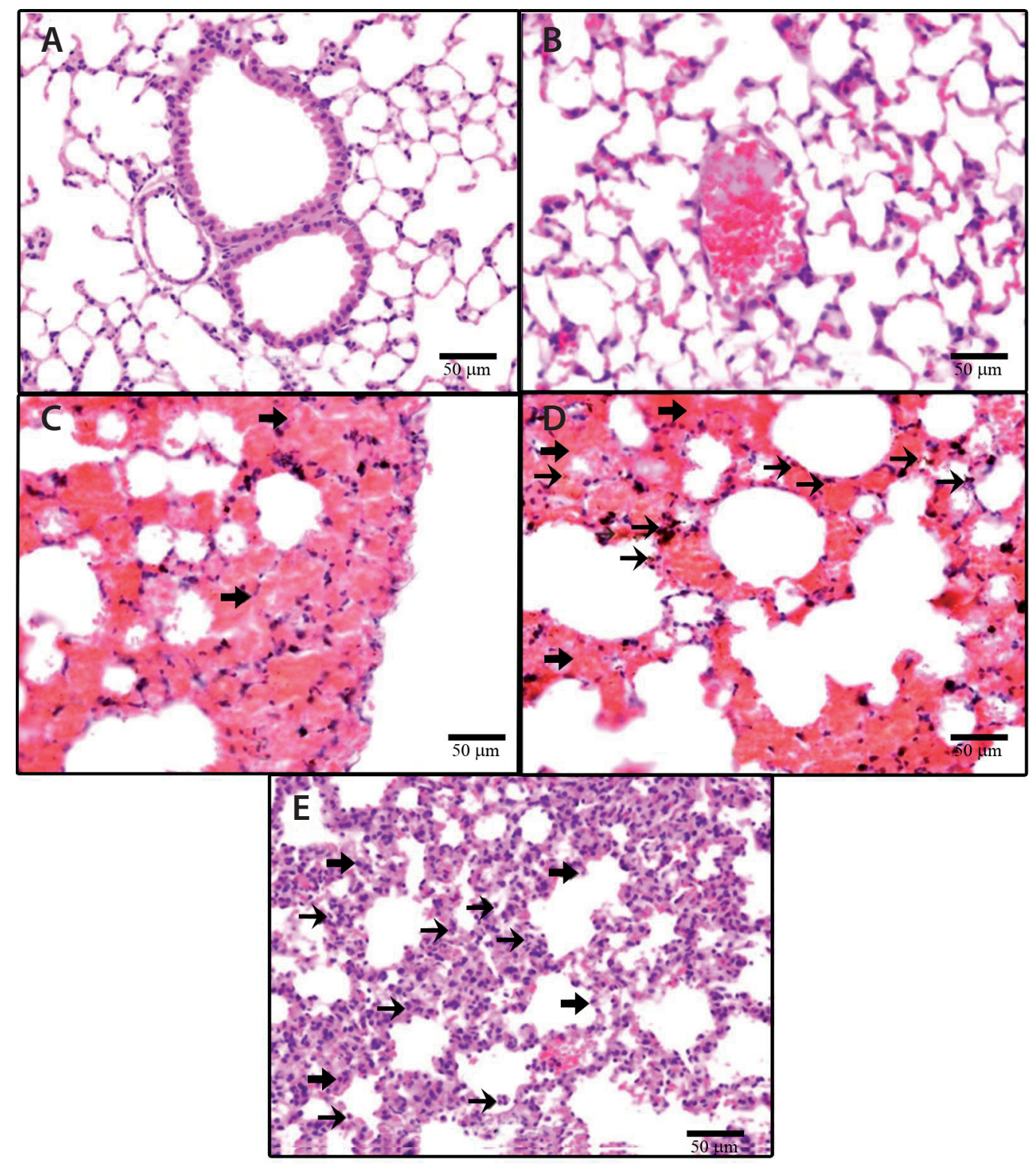

Figure 6. A. Lung section of control group shows normal lung morphology and architecture. B. Lung section of nicotine-treated group shows intra-vascular thrombosis. C. Lung section of nicotine-treated group shows hemorrhagic infarction of the lung with necrosis of inter-alveolar septae (thick arrows) and filling the alveoli with hemorrhage. D. Many hemosiderin-laden macrophages (thin arrows) are seen in the infarcted area. E. Widening of the inter-alveolar septae with inflammatory cells infiltration which consists of neutrophil polymorphs (thin arrows) and mononuclear cells (thick arrows). (All images: H\&E). 
of nicotine, at a concentration found in smokers and users of tobacco products, selectively impairs eNOS-dependent responses of cerebral arterioles. This impairment is via increase in superoxide anion production - an oxidative stress status. Our findings show decrease in SOD level as an indication of oxidative stress and increase in LDH level as indication of tissue damage caused by nicotine. The hepatocellular damage and that of the alveoli in the lungs are in line with previous studies which reported also depletion of SOD and elevation of $\mathrm{LDH}$ in sub-cutaneous nicotine treatment for 22 weeks (2.5 mg/kg body weight) in rats (Reddy et al. 2009).

WBC estimation in the nicotine-treated group showed a rise, although insignificant, which may be the sign of systemic inflammation in mice. Previous study (Roethig et al. 2010) in concordance with our results showed, on human consuming nicotine by smoking, a similar increase in WBC varying from $5-30 \%$. However, the increased levels of RBC in the smokers as shown in the same report, was not evident

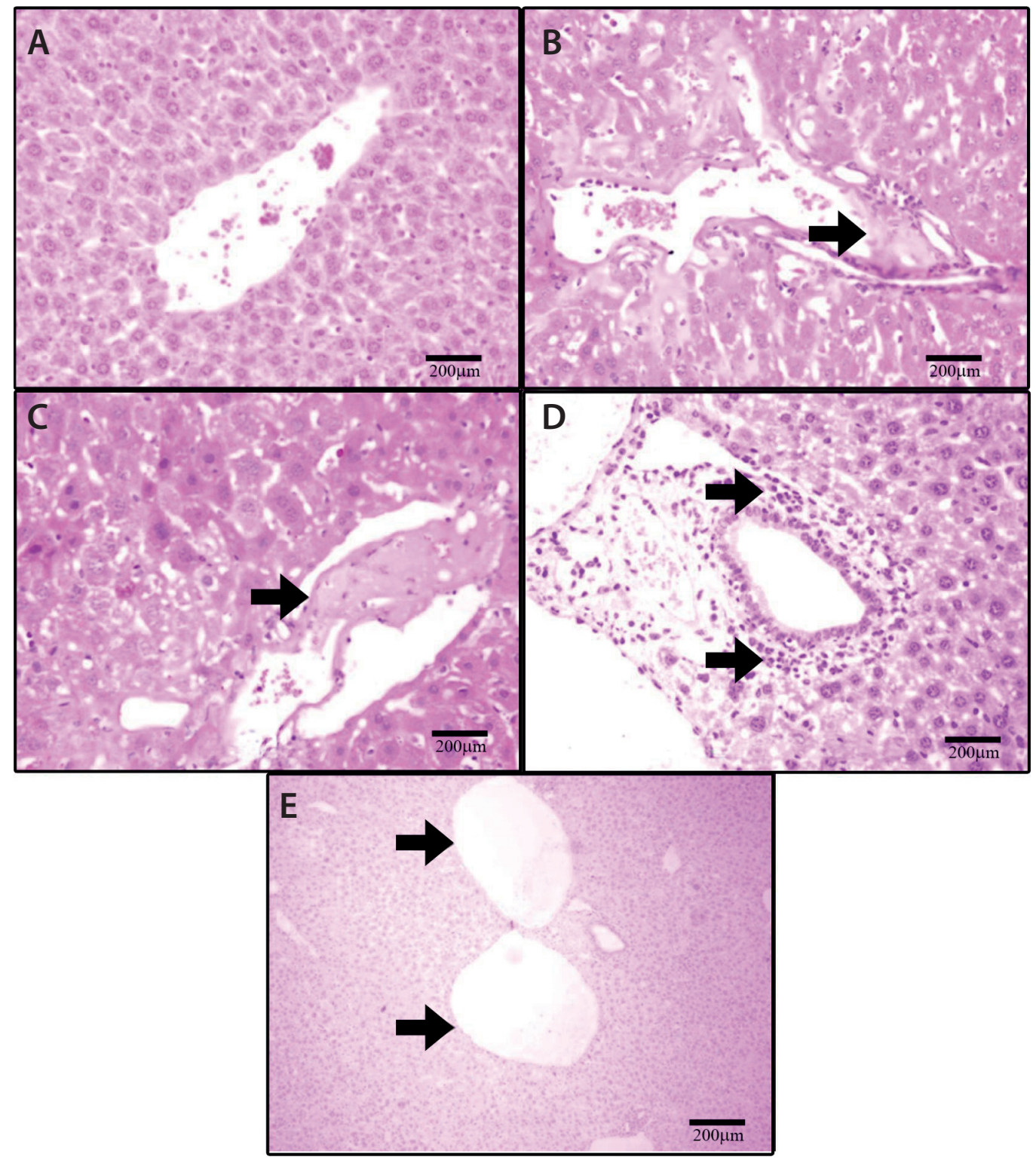

Figure 7. A. Liver section of control group shows normal liver morphology and architecture. B., C. Liver sections of nicotine-treated group show a thrombus (thick arrows) involving the central hepatic venule which is strictly adherent to the endothelial surface of the affected terminal hepatic venule. D. Liver section of nicotine-treated group shows portal inflammation (thick arrows). E. Liver section of nicotine-treated group shows widening and dilatation of terminal hepatic venule. (All images: H\&E). 
in our study, which may be partly due to the limitation of duration of the treatment in our study.

Nicotine induced acute changes in the liver enzymes of male Wistar rats accompanied with significant rise in ALT, AST and BUN (Bekairi et al. 1987), which was also reflected in this present study. The rise in the AST and ALT after the nicotine is well attributed to the damage caused by systemically treated nicotine thereby inducing the release of AST and ALT from the liver. However, the multi center clinical study on chronic smokers in men in London showed no significance changes in AST or ALT activities with smoking (Whitehead et al. 1996). Liver enzyme GGT is an important predictor of incidence and mortality of various diseases in particular to cardiovascular risk was not influenced by nicotine treatment in our study. This is in concordance with a large population-based cohort study in Germany which showed that the smoking nicotine do not affect GGT (Breitling et al. 2009).

An insignificant rise in the plasma levels of the creatinine due to nicotine treatment was observed by us what is in line with the observation of other groups (Sener et al. 2005). However, Arany et al. (2011) found that chronic nicotine exposure increased the extent of renal injury which may be attributed to their prolonged treatment for 4 weeks. Similarly, measurement of BUN showed no significant changes which is in contrary to the finding of Sener et al. (2005) who show a significant increase in the BUN values. Interestingly, nicotine by itself produced a less profound effect on BUN than to its combination treatment with alcohol (Bekairi et al. 1987). Moreover, the negligible changes in kidney morphology after treatment with nicotine are in support with our creatinine and BUN analysis.

The hemorrhagic infarction with necrosis in the lung due to the intra-vascular thrombosis, and the inflammatory endothelial lining with the dilatation of hepatic vasculature caused by nicotine demonstrate that the systemic administration of nicotine could possess toxicity on both the lung and the liver. Numerous mechanisms for an association between cigarette smoking and accelerated progression of liver fibrosis have been reported including tissue hypoxia, iron accumulation, tissue inflammation, oxidative stress and lipid peroxidation (Bataller 2006). However, the uninfluenced heart morphology suggests no damage caused by nicotine treatment in heart muscle.

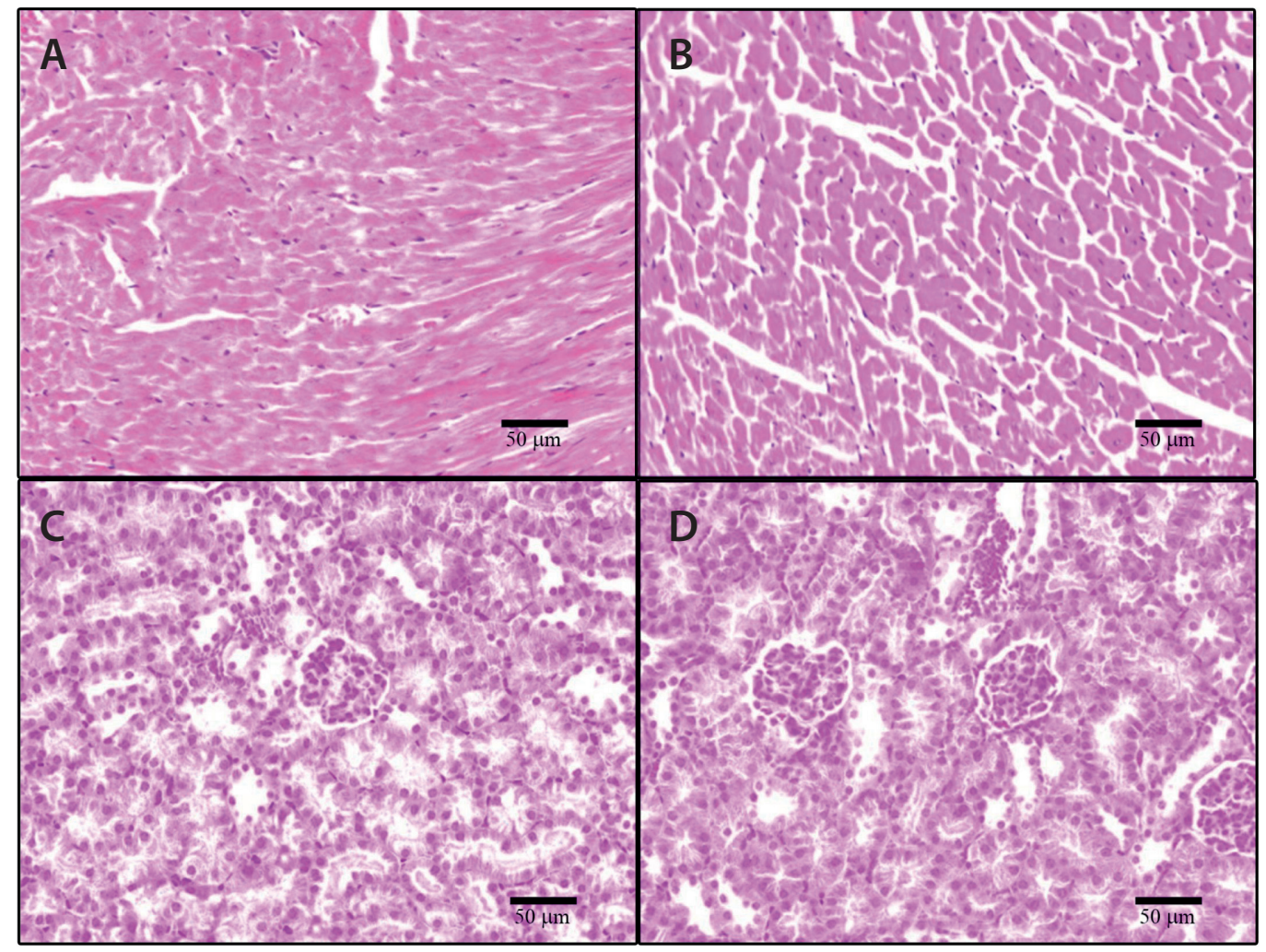

Figure 8. A. Heart section of control group shows normal heart morphology and architecture. B. Heart section of nicotine-treated group shows normal heart morphology and architecture. C. Kidney section of control group shows normal morphology and architecture. D. Kidney section of nicotine-treated group shows normal morphology and architecture. (All images: H\&E). 


\section{Conclusion}

In summary, our study suggests that nicotine increases the risk for thrombosis and cytotoxicity that leads to worsening of the hepatic and lung damages. Further investigations are in headway with varying degree of doses of nicotine which is indispensable for understanding more about the dynamics behind nicotine-induced subacute toxicity.

Acknowledgement. We would like to thank the Faculty of Medicine and Health Sciences, UAE University, Al Ain for funding this project. The authors would like to thank Mr. Sarabjit Singh, Mrs. Manjusha Sudhadevi and Mrs. Shaheen Zia for their technical assistance.

Authors' contributions. M. A. F., A. N., M. Y. H. designed the experimental protocol, the study concept and the research design; S. D., A. N. drafted and revised the manuscript; S. A., S. D., M. S., J. Y. performed the experiments. M. A. F., A. N., S. D., S. A. interpreted the data. All authors read and approved the final version of the manuscript.

There is no conflict of interest.

\section{References}

Al Dhaheri A. H., El-Sabban F., Fahim M. A. (1995): Chronic lead treatment accelerates photochemically induced platelet aggregation in cerebral microvessels of mice, in vivo. Environ. Res. 69, 51-58 http://dx.doi.org/10.1006/enrs.1995.1024

Arany I., Grifoni S., Clark J. S., Csongradi E., Maric C., Juncos L. A. (2011): Chronic nicotine exposure exacerbates acute renal ischemic injury. Am. J. Physiol. Renal. 301, F125-133. http://dx.doi.org/10.1152/ajprenal.00041.2011

Arrick D. M., Mayhan W. G. (2007): Acute infusion of nicotine impairs nNOS-dependent reactivity of cerebral arterioles via an increase in oxidative stress. J. Appl. Physiol. 103, 2062-2067 http://dx.doi.org/10.1152/japplphysiol.00411.2007

Bataller R. (2006): Time to ban smoking in patients with chronic liver diseases. Hepatology (Baltimore, Md.) 44, 1394-1396 http://dx.doi.org/10.1002/hep.21484

Bekairi A. M., Abulaban F. S., Tariq M., Parmar N. S., Ageel A. M. (1987): Studies on ethanol and/or nicotine induced acute changes in the levels of plasma amino acids and other biochemical parameters of male Wistar rats. Alcohol Drug. Res. 7, 471-479

Bernhard D., Pfister G., Huck C. W., Kind M., Salvenmoser W., Bonn G. K., Wick G. (2003): Disruption of vascular endothelial homeostasis by tobacco smoke: impact on atherosclerosis. FASEB J. 17, 2302

Breitling L. P., Raum E., Müller H., Rothenbacher D., Brenner H. (2009): Synergism between smoking and alcohol consumption with respect to serum gamma-glutamyltransferase. Hepatology 49, 802-808 http://dx.doi.org/10.1002/hep.22727
Cirillo P., De Rosa S., Pacileo M., Gargiulo A., Leonardi A., Angri V., Formisano S., Chiariello M. (2006): Nicotine induces tissue factor expression in cultured endothelial and smooth muscle cells. J. Thromb. Haemost. 4, 453-458 http://dx.doi.org/10.1111/j.1538-7836.2006.01741.x

Elliott B. M., Faraday M. M., Phillips J. M., Grunberg N. E. (2004): Effects of nicotine on elevated plus maze and locomotor activity in male and female adolescent and adult rats. Pharmacol. Biochem. Behav. 77, 21-28 http://dx.doi.org/10.1016/j.pbb.2003.09.016

Fahim M. A., Nemmar A., Singh S., Hassan M. Y. (2011): Antioxidants aleviate nicotine-induced platelet aggregation in cerebral arterioles of mice in vivo. Physiol. Res. 60, 695-700

Fang Q., Sun H., Mayhan W. G. (2003): Impairment of nitric oxide synthase-dependent dilatation of cerebral arterioles during infusion of nicotine. Am. J. Physiol. Heart Circ. Physiol. 284, H528-534

Fang Q., Sun H., Arrick D. M., Mayhan W. G. (2006): Inhibition of NADPH oxidase improves impaired reactivity of pial arterioles during chronic exposure to nicotine. J. Appl. Physiol. 100, 631-636 http://dx.doi.org/10.1152/japplphysiol.00975.2005

Folts J. D., Bonebrake F. C. (1982): The effects of cigarette smoke and nicotine on platelet thrombus formation in stenosed dog coronary arteries: inhibition with phentolamine. Circulation 65, 465-470 http://dx.doi.org/10.1161/01.CIR.65.3.465

Foo L. C., Roshidah I., Aimy M. B. (1991): Platelets of habitual smokers have reduced susceptibility to aggregating agent. Thromb. Haemost. 65, 317-319

Hanna S. T. (2006): Nicotine effect on cardiovascular system and ion channels. J. Cardiovasc. Pharmacol. 47, 348-358

Hioki H., Aoki N., Kawano K., Homori M., Hasumura Y., Yasumura T., Maki A., Yoshino H, Yanagisawa A., Ishikawa K. (2001): Acute effects of cigarette smoking on platelet-dependent thrombin generation. Eur. Heart J. 22, 56 http://dx.doi.org/10.1053/euhj.1999.1938

Hung J, Lam J. Y., Lacoste L., Letchacovski G. (1995): Cigarette smoking acutely increases platelet thrombus formation in patients with coronary artery disease taking aspirin. Circulation 92, 2432-2436 http://dx.doi.org/10.1161/01.CIR.92.9.2432

Iranloye B. O., Bolarinwa A. F. (2009): Effect of nicotine administration on weight and histology of some vital visceral organs in female albino rats. Niger. J. Physiol. Sci. 24, 7-12 http://dx.doi.org/10.4314/njps.v24i1.46374

Lau P. P., Li L., Merched A. J., Zhang A. L., Ko K. W., Chan L. (2006): Nicotine induces proinflammatory responses in macrophages and the aorta leading to acceleration of atherosclerosis in lowdensity lipoprotein receptor(-/-) mice. Arterioscler. Thromb. Vasc. Biol. 26, 143-149 http://dx.doi.org/10.1161/01.ATV.0000193510.19000.10

Lee J., Cooke J. P. (2011): The role of nicotine in the pathogenesis of atherosclerosis. Atherosclerosis 215, 281-283 http://dx.doi.org/10.1016/j.atherosclerosis.2011.01.003

Lindenblatt N., Platz U., Hameister J., Klar E., Menger M. D., Vollmar B. (2007): Distinct effects of acute and chronic nicotine application on microvascular thrombus formation and 
endothelial function in male and female mice. Langenbecks Arch. Surg. 392, 285-295

http://dx.doi.org/10.1007/s00423-007-0173-6

Matta S. G., Balfour D. J., Benowitz N. L., Boyd R. T., Buccafusco J. J., Caggiula A. R., Craig C. R., Collins A. C., Damaj M. I., et al. (2006): Guidelines on nicotine dose selection for in vivo research. Psychopharmacology (Berl) 190, 269-319 http://dx.doi.org/10.1007/s00213-006-0441-0

Miller K., Hudspith B., Cunninghame M., Prescott C., and Meredith C. (1996): Effect of cigarette smoke exposure on biomarkers of lung injury in the rat. Inhal. Toxicol. 8, 803-817 http://dx.doi.org/10.3109/08958379608995212

Nemmar A., Hoet P. H., Vandervoort P., Dinsdale D., Nemery B., Hoylaerts M. F. (2007): Enhanced peripheral thrombogenicity after lung inflammation is mediated by platelet-leukocyte activation: role of P-selectin. J. Thromb. Haemost. 5, 1217-1226 http://dx.doi.org/10.1111/j.1538-7836.2007.02557.x

Nemmar A., Al-Salam S., Dhanasekaran S., Sudhadevi M., Ali B. H. (2009): Pulmonary exposure to diesel exhaust particles promotes cerebral microvessel thrombosis: Protective effect of a cysteine prodrug 1-2-oxothiazolidine-4-carboxylic acid. Toxicology 263, 84-92 http://dx.doi.org/10.1016/j.tox.2009.06.017

Nemmar A., Al-Salam S., Zia S., Dhanasekaran S., Shudadevi M., Ali B. H. (2010): Time-course effects of systemically administered diesel exhaust particles in rats. Toxicol. Lett. 194, 58-65 http://dx.doi.org/10.1016/j.toxlet.2010.02.001

Nemmar A., Al-Salam S., Zia S., Marzouqi F., Al-Dhaheri A., Subramaniyan D., Dhanasekaran S., Yasin J., Ali B. H., Kazzam E. E. (2011): Contrasting actions of diesel exhaust particles on the pulmonary and cardiovascular systems and the effects of thymoquinone. Br. J. Pharmacol. 164, 1871-1882 http://dx.doi.org/10.1111/j.1476-5381.2011.01442.x

Niki E. (2004): Antioxidants and atherosclerosis. Biochem. Soc. Trans. 32, 156-159 http://dx.doi.org/10.1042/BST0320156

Prandoni P., Bilora F., Marchiori A., Bernardi E., Petrobelli F., Lensing A. W., Prins M. H., Girolami A. (2003): An association between atherosclerosis and venous thrombosis. N. Engl. J. Med. 348, 1435-1441 http://dx.doi.org/10.1056/NEJMoa022157

Rahman M. M., Laher I. (2007): Structural and functional alteration of blood vessels caused by cigarette smoking: an overview of molecular mechanisms. Curr. Vasc. Pharmacol. 5, 276-292 http://dx.doi.org/10.2174/157016107782023406

Reddy B. V., Sundari J. S., Balamurugan E., Menon V. P. (2009): Prevention of nicotine and streptozotocin treatment induced circulatory oxidative stress by bis-1,7-(2-hydroxyphenyl)hepta-1,6-diene-3,5-dione in diabetic rats. Mol. Cell. Biochem. 331, 127-133 http://dx.doi.org/10.1007/s11010-009-0150-1

Renaud S., Blache D., Dumont E., Thevenon C., Wissendanger T. (1984): Platelet function after cigarette smoking in relation to nicotine and carbon monoxide. Clin. Pharmacol. Ther. 36, 389-395 http://dx.doi.org/10.1038/clpt.1984.193

Roethig H. J., Koval T., Muhammad-Kah R., Jin Y., Mendes P., Unverdorben M. (2010): Short term effects of reduced exposure to cigarette smoke on white blood cells, platelets and red blood cells in adult cigarette smokers. Regul. Toxicol. Pharmacol. 57, 333-337

http://dx.doi.org/10.1016/j.yrtph.2010.04.005

Sabha M., Tanus-Santos J. E., Toledo J. C., Cittadino M., Rocha J. C., Moreno H. Jr. (2000): Transdermal nicotine mimics the smoking-induced endothelial dysfunction. Clin. Pharmacol. Ther. 68, 167-174 http://dx.doi.org/10.1067/mcp.2000.108851

Sener G., Sehirli O., Ipçi Y., Cetinel S., Cikler E., Gedik N., Alican I. (2005). Protective effects of taurine against nicotine-induced oxidative damage of rat urinary bladder and kidney. Pharmacology 74, 37-44 http://dx.doi.org/10.1159/000083245

Siess W., Lorenz R., Roth P., Weber P. C. (1982): Plasma catecholamines, platelet aggregation and associated thromboxane formation after physical exercise, smoking or norepinephrine infusion. Circulation 66, 44-48 http://dx.doi.org/10.1161/01.CIR.66.1.44

Singh U., Jialal I. (2006): Oxidative stress and atherosclerosis. Pathophysiology 13, 129-142 http://dx.doi.org/10.1016/j.pathophys.2006.05.002

Soeda J., Morgan M., McKee C., Mouralidarane A., Lin C., Roskams T., Oben J. A. (2012): Nicotine induces fibrogenic changes in human liver via nicotinic acetylcholine receptors expressed on hepatic stellate cells. Biochem. Biophys. Res. Commun. 417, 17-22 http://dx.doi.org/10.1016/j.bbrc.2011.10.151

Steiner A. A., Oliveira D. L., Roberts J. L., Petersen S. R., Romanovsky A. A. (2008): Nicotine administration and withdrawal affect survival in systemic inflammation models. J. Appl. Physiol. 105, 1028-1034 http://dx.doi.org/10.1152/japplphysiol.90619.2008

Steiner M. (1999): Vitamin E, a modifier of platelet function: rationale and use in cardiovascular and cerebrovascular disease. Nutr. Rev. 57, 306-309 http://dx.doi.org/10.1111/j.1753-4887.1999.tb06903.x

Whitehead T. P., Robinson D., Allaway S. L. (1996): The effects of cigarette smoking and alcohol consumption on serum liver enzyme activities: a dose-related study in men. Ann. Clin. Biochem. 33, 530 http://dx.doi.org/10.1177/000456329603300607

Yildiz D., Ercal N., Armstrong D. W. (1998): Nicotine enantiomers and oxidative stress. Toxicology 130, 155-165 http://dx.doi.org/10.1016/S0300-483X(98)00105-X

Yong T., Zheng M. Q., Linthicum D. S. (1997): Nicotine induces leukocyte rolling and adhesion in the cerebral microcirculation of the mouse. J. Neuroimmunol. 80, 158-164 http://dx.doi.org/10.1016/S0165-5728(97)00151-3

Received: August 14, 2013

Final version accepted: February 24, 2014 